\title{
RECURSIVE FUNCTIONS OF ONE VARIABLE
}

\author{
JULIA ROBINSON
}

We say that the function $F$ is obtained by general recursion from $A, B, M$, and $N$ if

(i) $F A=M, F B=N F$ and

(ii) every natural number $n$ belongs to $\mathbb{R}\left(B^{k} A\right)$ for some $k \geqq 0$.

If $F$ satisfies (i), then $F\left(B^{k} A t\right)=N^{k} M t$. If $A$ and $B$ satisfy (ii), then every natural number $n$ is $B^{k} A t$ for some $k$ and $t$. Hence $F$ is uniquely determined. Furthermore if $A, B, M$, and $N$ are computable functions, then given $n$, we can obtain $k$ and $t$ effectively so $F$ is computable.

Condition (ii) is satisfied if $A$ is the zero function $O$ and $B$ is the successor function $S$. There is a function obtained by general recursion from $O, S, M$, and $N$ if and only if $M$ is a constant function, say $M=S^{m} O$. Then $F t=N^{t} m$. Thus, iteration is included in general recursion.

If $G$ is a permutation, then $F=G^{-1}$ can be obtained by general recursion from $G S, G O, S$, and $O$ since $F G S=S, F G O=O F$, and every $n$ belongs to $\mathcal{R}\left((G O)^{k} G S\right)$ for $k=0$ or $k=1$. More generally, if $F$ is determined by $F G=H$ where $G$ assumes all values, then $F$ is obtained by general recursion from $G S, G O, H S$, and $H O$ since $F G S=H S$, $F G O=H O F$, and the side condition holds as before.

THEOREM. Every (general) recursive function of one variable can be obtained from $O$ and $S$ by repeated compositions and general recursions from previously defined functions.

We need to prove a lemma first. Let $J(x, y)$ be a one-to-one mapping of the set of ordered pairs of natural numbers onto the set of natural numbers and let $K$ and $L$ be the inverse functions so $K J(x, y)$ $=x$ and $L J(x, y)=y$. Any such function $J$ will be called a pairing function.

Lemma. Every primitive recursive function of one variable can be obtained from $O, S, K$, and $L$ by repeatedly constructing a new function $F$ from previously obtained functions $A$ and $B$ by composition $(F=A B)$, pairing $(F=J(A, B))$, or general recursion of the form $F J(I, O)=A$ and $F J(K, S L)=B F$.

Received by the editors April 1, 1967. 
REMARK. The general recursion of the lemma always defines a function. In fact, for every $n$, there is a unique pair $r, s$ with $n=J(r, s)$, so $F n=B^{s} A r$.

Proof. If $J, K$, and $L$ are adjoined to the usual initial functions in the standard definition of primitive recursive functions, then recursion can be replaced by iterations of the form

$$
G(x, 0)=A x, \quad G(x, S y)=B G(x, y) .
$$

(See R. M. Robinson [3, p. 932].) Since $J$ is an initial function, we can replace this iteration scheme by the general recursion of the lemma. Namely, if $F$ is defined by $F J(I, O)=A$ and $F J(K, S L)=B F$, then the function $G$ defined by (1) is $F J(x, y)$. Hence every primitive recursive function is obtained from the usual initial functions together with $J, K$, and $L$ by general recursion of the form in the lemma and substitution. To obtain functions of one variable by substitution, it is always sufficient to use substitution only to define functions of one variable. The only initial function of more than one variable which yields new functions of one variable by substitution is $J$. Hence besides compositions of functions of one variable, we must allow $F=J(A, B)$ to be constructed from previously obtained functions $A$ and $B$. Hence all primitive recursive functions of one variable are obtained from $O, I, S, K$, and $L$ by compositions, pairings, and general recursions of the form given in the lemma. Since $I=J(K, L)$, we can omit $I$ from the initial functions. (This proof closely parallels the proof given by R. M. Robinson in $\$ 7$ of [3]. A more detailed discussion will be found there.)

Proof of the Theorem. Let $R$ be the class of functions obtainable from $O$ and $S$ by composition and general recursion. We will show first that every primitive recursive function belongs to $R$.

Suppose that $J$ is a pairing function such that $J(I, O)$ and $J(K, S L)$ belong to $R$. Then $I, K$, and $L$ are in $R$ as we see by the general recursions:

$$
\begin{aligned}
I O & =O, & & I S=S I ; \\
K J(I, O) & =I, & & K J(K, S L)=I K ; \\
L J(I, O) & =O, & & L J(K, S L)=S L .
\end{aligned}
$$

In order to use the lemma, we need to show that $R$ is closed under pairing. Let $F^{*}=J(K, F L)$ for each $F$. We will show by induction that $R$ is closed under the ${ }^{*}$-operation.

For the initial functions $O$ and $S$, we have

$$
O^{*}=J(K, O L)=J(I, O) K, \quad S^{*}=J(K, S L),
$$

so $O^{*}$ and $S^{*}$ are both in $R$. 
Furthermore,

$$
A^{*} B^{*}=J(K, A L) J(K, B L)=J(K, A B L)=(A B)^{*} .
$$

Hence if $F=A B$ and $A^{*}, B^{*} \in R$, then $F^{*}$ is in $R$.

Suppose $F$ is obtained from $A, B, M$, and $N$ by general recursion $F A=M, F B=N F$, and $A^{*}, B^{*}, M^{*}$, and $N^{*}$ belong to $R$. Then

$$
F^{*} A^{*}=(F A)^{*}=M^{*}, \quad F^{*} B^{*}=(F B)^{*}=(N F)^{*}=N^{*} F^{*} .
$$

We still must check that $A^{*}$ and $B^{*}$ satisfy (ii) given that $A$ and $B$ do. For every $n$, there are $r$ and $s$ so that $n=J(r, s)$. By hypothesis $s=B^{k} A t$ for some $k$ and $t$, so $\left(B^{*}\right)^{k} A^{*} J(r, t)=J\left(r, B^{k} A t\right)=J(r, s)=n$. Hence $n$ belongs to $R\left(\left(B^{*}\right)^{k} A^{*}\right)$ and (ii) holds. Therefore if $F$ is in $R$, so also is $F^{*}$.

Next we will show that if $A$ and $B$ are in $R$, then $J(A, B)$ is in $R$. Now

$$
J(A, B)=J(L, K) A^{*} J(L, K) B^{*} J(I, I) .
$$

To check this, notice that

$J(L, K) A^{*}=J(L, K) J(K, A L)=J(A L, K), \quad J(L, K) B^{*}=J(B L, K)$.

Hence

$$
\begin{aligned}
J(L, K) A^{*} J(L, K) B^{*} J(I, I) & =J(A L, K) J(B L, K) J(I, I) \\
& =J(A K, B L) J(I, I)=J(A, B) .
\end{aligned}
$$

Both $J(L, K)$ and $J(I, I)$ are in $R$ as we see from the following series of definitions by general recursions:

$$
\begin{aligned}
J(O, I) O & =J(I, O) O, \quad J(O, I) S=J(K, S L) J(O, I) ; \\
J(S K, L) J(I, O) & =J(I, O) S, \quad J(S K, L) J(K, S L)=J(K, S L) J(S K, L) ; \\
J(L, K) J(I, O) & =J(O, I), \quad J(L, K) J(K, S L)=J(S K, L) J(L, K) ; \\
J(I, I) O & =J(I, O) O, \quad J(I, I) S=J(S K, L) J(K, S L) J(I, I) .
\end{aligned}
$$

We have already seen that (ii) holds in each of these case, so the definitions are legitimate. Hence $J(A, B)$ is in $R$.

Hence by the lemma, every primitive recursive function belongs to $R$ provided there is a pairing function $J$ such that $J(I, O)$ and $J(K, S L)$ are in $R$. One such $J$ is given by $J(x, y)=2^{y}(2 x+1)-1$. For this $J, J(x, 0)=2 x$ and $J(x, S y)=2 J(x, y)+1$. Hence $J(I, O)$ is the double function $D$ and $J(K, S L)$ is $S D$. Of course $D$ belongs to $R$ since it is defined by the general recursion: $D O=O$ and $D S=S S D$. Therefore every primitive recursive function belongs to $R$. 
Finally, if $F$ is recursive and $J$ is the function given above (or any recursive pairing function) then $\{y: y=J(x, F x)\}$ is a recursive set and hence the range of some primitive recursive function $G$. So $F$ is defined by the equation $F K G=L G$, where $K G$ assumes all values. We have already seen that $F$ is obtained by the general recursion $F K G S=L G S$ and $F K G O=L G O F$. Hence every recursive function of one variable is in $R$.

A functional equation is an equation of the form

$$
A_{1} \cdots A_{k}=B_{1} \cdots B_{l} .
$$

We say that the equation $\alpha=\beta$ is derivable from a system $\Sigma$ of functional equations if there is a chain of equations $\alpha_{1}=\cdots=\alpha_{n}(n \geqq 1)$ such that $\alpha_{1}=\alpha, \alpha_{n}=\beta$, and for each $t, \alpha_{t+1}$ is obtained from $\alpha_{t}$ by replacing a part $\gamma$ of $\alpha_{t}$ by $\delta$ where $\gamma=\delta$ or $\delta=\gamma$ belongs to $\Sigma$.

A system $\Sigma\left(S, F, U_{1}, \cdots\right)$ of functional equations defines a particular function if $\Sigma$ has a unique solution in which $S$ is the successor function, and in this solution $F$ is the required function. Even though $\Sigma$ defines $F$, it may not provide any way to compute the values of $F$. Indeed, every hyperarithmetical function can be defined by some system of functional equations [2]. Hence in general there is no way at all to compute a function defined by a system of functional equations.

ExAmple. The equations

$$
D O=O, \quad D S=S S D
$$

define both the zero function $O$ and the double function $D$. The second equation gives $D x=2 x+D 0$. Hence the only possible fixed point for $D$ is at $x=0$. Therefore the first equation implies $R(O)=\{0\}$. Hence $O$ is identically 0 and $D x=2 x$. Furthermore, it is easily seen that every equation of the form $D S^{k} O=S^{2 k} O$ can be derived from (2). (Here $S^{k}$ is an abbreviation for $S \cdots S$ with $k S$ 's.) However, the equation $O O=O$ cannot be derived from (2), since any equation derived from (2) will have the same number of $O$ 's on each side.

On the other hand, all equations of the form $O S^{k} O=O$ can be derived from

$$
O O=O, \quad O S=O .
$$

But (3) does not define the function $O$. Indeed, any constant function can be substituted for $O$ in (3) and it will still be satisfied. However, the system consisting of (2) and (3) does define $O$ and $D$, and from it, every equation of the forms $O S^{k} O=O, D S^{k} O=S^{2 k} O$ can be derived. 
CoRollary 1. A function of one variable is recursive if and only if there is a system $\Sigma\left(S, O, F, U_{1}, \cdots\right)$ of functional equations such that

(i) $\Sigma$ has a unique solution in which $S$ is the successor function and $O$ is the zero function. In this solution, $F$ is the given function.

(ii) For all natural numbers $n$ and $m$, the equation $F S^{n} O=S^{m} O$ is derivable from $\Sigma$ if and only if $F n=m$.

REMARks. The "only if" part of (ii) follows from (i) since $F$ satisfies $\Sigma$ with $S$ and $O$ the successor and zero functions, it must also satisfy any equation derived from $\Sigma$. If (ii) holds, we say the values of $F$ can be derived from $\Sigma$. The theorem corresponding to Corollary 1 for functions of any number of variables was proved by Kreisel and Tait [1].

Proof. Start with the equations $O O=O, O S=O$.

To obtain $\Sigma$ for a recursive function $F$, adjoin a sequence of equations corresponding to successive definitions of new functions by compositions and general recursions from $O, S$, and functions already defined, ending with a definition of $F$. This is possible by the theorem. Clearly, $\Sigma$ satisfies (i) since each function introduced is uniquely determined by its defining equations from earlier functions.

We will show now by induction that the values of $F$ can be derived from $\Sigma$. Suppose $G$ is $O$ or $S$. The values of $O$ are derivable from the first two equations. The values of the successor function are given by identities $S\left(S^{k} O\right)=S^{k+1} O$.

Suppose $G=A B$ is in $\Sigma$ and the values of $A$ and $B$ can be derived from $\Sigma$. Then we can derive the values of $G$ since

$$
G S^{n} O=A B S^{n} O=A S^{B n} O=S^{A B n} O=S^{G n} O .
$$

Here $B n, A B n$, and $G n$ are natural numbers indicating the number of $S$ 's in the expression.

Suppose $G A=M$ and $G B=N G$ are in $\Sigma$, every number $n$ is $B^{k} A t$ for some $k$ and $t$, and the values of $A, B, M$, and $N$ are derivable from $\Sigma$. Then the values of $G$ are derivable, since

$$
\begin{aligned}
G S^{n} O=G B^{k} A S^{t} O & =N^{k} G A S^{t} O=N^{k} M S^{t} O=N^{k} S^{M}{ }^{t} O \\
& =S^{N^{k} M t} O=S^{G n} O .
\end{aligned}
$$

Conversely, if $\Sigma$ satisfies (ii), then $F$ is computable. We simply systematically derive all possible equations from $\Sigma$. For each $n$, we will eventually derive an equation of the form $F S^{n} O=S^{m} O$ and conclude that $F n=m$. Hence by Church's Thesis, $F$ is recursive. A mathematical proof that $F$ is recursive can be given in the standard way. 
Corollary 2 (Post, Turing 1948). The word problem for semigroups (associative systems) is recursively unsolvable.

Proof. Let $F$ be a recursive function whose range is not recursive and let $\Sigma$ satisfy Corollary 1 for this $F$. Let $\Sigma^{\prime}$ be obtained from $\Sigma$ by adjoining the single equation $G F=O$.

Then $G S^{n} O$ will be derivable from $\Sigma^{\prime}$ if and only if $n$ is in $R(F)$. The function $G_{0}$ given by

$$
\begin{aligned}
G_{0} n & =0 & & \text { if } n \in \mathcal{R}(F), \\
& =1 & & \text { otherwise }
\end{aligned}
$$

satisfies $\Sigma^{\prime}$ and hence $G S^{n} O=O$ cannot be derived from $\Sigma^{\prime}$ if $n \notin R(F)$. Since $R(F)$ is not recursive, there is no general method of telling whether or not an arbitrary equation of the form $\alpha=O$ is derivable from $\Sigma^{\prime}$.

\section{REFERENCES}

1. Georg Kreisel and William W. Tait, Finite definability of number-theoretic functions and parametric completeness of equational calculi, Z. Math. Logik Grundlagen Math. 7 (1961), 28-38.

2. Julia Robinson, An introduction to hyperarithmetical functions, J. Symbolic Logic 32 (1967), 325-342.

3. Raphael M. Robinson, Primitive recursive functions, Bull. Amer. Math. Soc. 53 (1947), 925-942.

University of California, Berkeley 